\title{
Diversidade de aves, mamíferos e répteis atropelados em região de floresta subtropical no sul do Brasil
}

\author{
Diversity of birds, mammals and reptiles roadkilled in a subtropical forest region in \\ southern Brazil
}

\author{
Angela Camila Deffaci, Vânia Patrícia da Silva, Marilia Teresinha Hartmann \\ e Paulo Afonso Hartmann. \\ Universidade Federal da Fronteira Sul - UFFS, Campus Erechim, RS, Brasil \\ cami.defffaci@hotmail.com; pollyvps@gmail.com; marilia.hartmann@gmail.com; hartmann.paulo@gmail.com
}

\begin{abstract}
Resumo
O objetivo deste estudo foi estimar as de espécies de aves, mamíferos e répteis atropelados em região de floresta subtropical e discutir quais fatores influenciam nos atropelamentos. Dados sobre os atropelamentos foram obtidos em dois trechos de $12 \mathrm{~km}$, percorridos de carro em velocidade de $50 \mathrm{~km} / \mathrm{h}$. Foram realizados 10 dias de amostragens por mês, de setembro de 2014 a fevereiro de 2015. Foi calculada a taxa de atropelamentos e a curva de acumulação de espécies. Estimou-se a riqueza de espécies e determinou-se a frequência de atropelamento de cada espécie. Foram encontrados 209 indivíduos atropelados de 45 espécies. A taxa de atropelamento foi de 0,14 ind./km/dia. Cinco espécies constam entre as mais atropeladas. As espécies com maior número de atropelamentos coincidem com as espécies classificadas como Constantes ou Acessórias. A taxa de atropelamento indica que nos períodos mais quentes do ano podem ser encontrados ao menos 50 animais atropelados por quilômetro nas rodovias avaliadas. A similaridade baixa e as diferenças na diversidade entre os dois trechos indicam possível influência da paisagem do entorno da rodovia. Rodovias que cortam áreas preservadas tendem a gerar impactos sobre espécies mais exigentes em relação ao uso de habitat.
\end{abstract}

Palavras - chave: Ecologia de estradas, Vertebrados, Impacto, Conservação.

\begin{abstract}
The aim of this study was to estimate the diversity of birds, mammals and reptiles roadkilled in a subtropical forest region in southern Brazil. Data on roadkill were obtained from two $12 \mathrm{~km}$ sections. The sections were covered by car in both directions, at an average speed of $50 \mathrm{~km} / \mathrm{h}$. Two surveys were carried out per month, lasting five days each, totalizing 10 days per month. The roadkill rate and the species accumulation curve was calculated. The species richness was estimated and the species most knocked down was determined. A total of 209 roadkill individuals were found, belonging to 45 species. The roadkill rate was 0.14 ind./Km/day. Five species are among the most roadkilled. The species with the highest number of roadkill coincide widely with the species classified as Constants or Accessory. The roadkill rates showed that at less 50 animals can be found roadkilled during the heat seasons in the evaluated roads. The low similarity and the differences between the two roads showed the possible influence the landscape in the diversity of fauna roadkilled. Roads that crossing preserved areas seems impact animals more exigent about the habitat use.
\end{abstract}

Keywords: Road ecology, Diversity, Environmental impact, Conservation. 


\section{Introdução}

Um dos impactos mais perceptíveis na fauna, gerados pela implantação e operação de rodovias, é a morte de animais silvestres causadas pela colisão com veículos (CLEVENGER et al., 2003; FORMAN et al., 2003; ANDREWS; GIBBONS, 2005; BENÍTEZ-LÓPEZ et al., 2010; VAN DER REE et al., 2015). Esta tem sido apontada como a principal causa de mortalidade de vertebrados por influência direta de atividades humanas no Brasil e no mundo (FORMAN; ALEXANDER, 1998; BAGER et al., 2016). Os atropelamentos geralmente ocorrem em função de a rodovia cortar o habitat de determinado táxon, o que interfere nos deslocamentos dos indivíduos. Aparentemente, alto volume de tráfego gera mais efeito evitação, com os animais mantendo-se distante da rodovia, enquanto rodovias de médio e baixo volume de tráfego parecem potencializar os atropelamentos (FORMAN; ALEXANDER, 1998; LAURANCE et al., 2009; FAHRIG; RYTWINSKIN, 2009).

A mortalidade gerada pelos atropelamentos pode ter efeitos importantes na demografia, pois pode afetar a densidade e a estrutura sexual, etária e genética das populações silvestres (FAHRIG et al., 1995; HUIJSER; BERGERS, 2000; GIBBS; STEEN, 2005). Mesmo uma baixa taxa de atropelamento pode representar uma perda significativa para populações raras ou ameaçadas de extinção (FORMAN; ALEXANDER, 1998). No entanto, o número de ocorrências de atropelamento de uma determinada espécie não significa, necessariamente, uma ameaça para a persistência da população, pois pode indicar uma elevada abundância local ou uma ampla distribuição espacial da população (CÁCERES et al., 2010).

Os atropelamentos de fauna silvestre normalmente se concentram em poucas espécies (COELHO et al., 2008; GRILO et al., 2009; HARTMANN et al., 2011, 2012; CUNHA et al., 2015), e as diferenças no número de atropelamentos podem estar relacionadas com as características comportamentais e ecológicas das espécies, como sexo, idade, respostas de evitação à estrada, capacidade de dispersão, densidade populacional e horário de atividade (JAEGER et al., 2005; FORD; FAHRIG, 2007; HARTMANN et al., 2011). As características da paisagem do entorno da estrada e as características da própria estrada, por influenciarem no comportamento e ecologia das espécies, também podem afetar na taxa de atropelamento (CLEVENGER et al., 2003; GOOSEM, 2007; COELHO et al., 2008). Em função disto, os fatores que determinam os atropelamentos variam dentro dos diversos grupos taxonômicos (FORMAN et al., 2003).

Estimar as espécies sujeitas aos atropelamentos em diferentes paisagens, assim como estabelecer qual proporção e as possíveis relações com a ecologia das espécies, contribui para o estabelecimento de medidas mitigadoras (ERRITOZOE et al., 2003; BAGER; ROSA, 2012). Entender os mecanismos envolvidos na relação entre empreendimentos rodoviários e a ecologia das populações e comunidades é de grande aplicabilidade e pode subsidiar a tomada de decisões no planejamento territorial e em estratégias de conservação (DRAMASTAD et al., 1996; FORMAN, 2004).

No Brasil, as colisões com veículos automotores representam uma parcela importante da perda local de biodiversidade, podendo gerar declínios em população de muitas espécies silvestres (e.g. TUMELEIRO et al., 2006; TURCI; BERNARDE, 2009; HARTMANN et al., 2011, 2012; CUNHA et al., 2015). O tamanho do país, a heterogeneidade de paisagens e as diferenças na diversidade de fauna de cada região dificultam encontrar padrões gerais. Neste sentido, amostragens locais ou regionais se mostram mais adequadas para o entendimento dos fatores responsáveis pelo atropelamento em cada região. O objetivo deste estudo foi estimar a diversidade de espécies de aves, mamíferos e répteis atropelados em uma região de floresta subtropical e discutir quais fatores podem influenciar nos atropelamentos.

\section{Métodos}

\section{Área de estudo}

O estudo foi realizado em duas rodovias na região norte do Estado do Rio Grande do Sul. A região está situada no limite sul do domínio morfoclimático do Bioma Mata Atlântica e integra a área da Reserva da Biosfera da Mata Atlântica. É caracterizada por uma vegetação ombrófila mista e clima subtropical úmido (AB'SABER; MARIGO, 2006). As rodovias amostradas foram: RS-331, entre os municípios de Erechim e Gaurama (T1) e RS-420, entre os municípios de Erechim e Aratiba (T2). As rodovias interligam o município de Erechim a municípios com aproximadamente 6.000 habitantes e de economia essencialmente agrícola (IBGE, 2010). O volume de tráfego é considerado baixo ( $<1.000$ veículos/dia) e caracterizado por automóveis, ônibus, caminhões e máquinas agrícolas (IBGE, 2010). Estruturalmente as rodovias são de pistas simples e pavimentadas, com acostamentos reduzidos (cerca de $80 \mathrm{~cm}$.), e limite de velocidade de $80 \mathrm{~km} / \mathrm{h}$.

A paisagem no entorno das duas rodovias é diferente quanto ao relevo, uso e ocupação do solo. O trecho T1 apresenta relevo de ondulação suave, escassos fragmentos florestais e predomínio de áreas agrícolas (Figura 1A). O trecho T2 apresenta relevo de ondulações acentuadas e predomínio de vegetação florestal na encosta dos morros, intercalada com pequenas áreas agrícolas nas áreas planas (Figura 1B). A temperatura média máxima na região é de $23,3^{\circ} \mathrm{C}$ e a média mínima é de $13,5^{\circ} \mathrm{C}$ com uma precipitação média de $1.607,2 \mathrm{~mm}$ por ano (dados referentes ao ano de 1999 a 2013; FEPAGRO, 2014). 

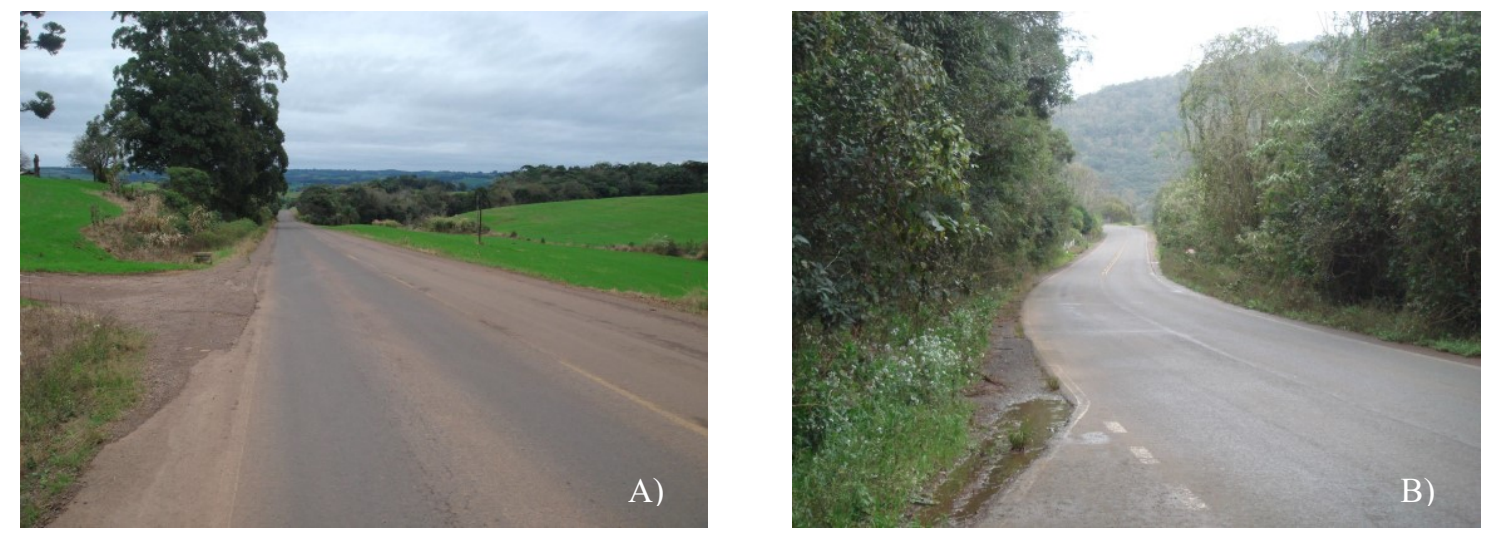

Figura 1 - Paisagem no entorno do Trecho T1 (RS-331), entre os municípios Erechim e Gaurama (A) e Trecho T2 (RS-420), entre os municípios de Erechim e Aratiba (B), Norte do Estado do Rio Grande Sul

Foto: Autores

\subsection{Coleta dos dados}

Para a coleta dos dados sobre os atropelamentos, equipes compostas por duas ou três pessoas, percorreram de carro ("driving surveys", LANGEN et al., 2007)

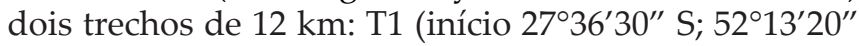
O; fim $27^{\circ} 35^{\prime} 26^{\prime \prime}$ S; $52^{\circ} 07^{\prime} 19^{\prime \prime}$ O) e T2 (início $27^{\circ} 36^{\prime} 16^{\prime \prime}$ S; $52^{\circ} 16^{\prime} 56^{\prime \prime}$ O; fim $27^{\circ} 32^{\prime} 05^{\prime \prime}$ S; $52^{\circ} 19^{\prime} 25^{\prime \prime}$ O). Os trajetos foram percorridos nos dois sentidos, no mesmo dia, a uma velocidade média de $50 \mathrm{~km} / \mathrm{h}$. Esta velocidade permite deteçãao de grande parte da diversidade e confere segurança durante os deslocamentos, embora animais de pequeno porte no acostamento possam não ser detectados (LANGEN et al., 2007; CUNHA et al., 2015). Foram realizados dois blocos de amostragens por mês, com duração de cinco dias cada e intervalo de 10 dias entre os blocos, totalizando 10 dias de amostragem por mês, em cada trecho. O primeiro bloco de amostragem de cada mês foi realizado no turno da manhã (entre 07h30min e 11:00 horas) e a segundo bloco no turno da tarde (entre 13:30 e 17:00 horas). As amostragens duraram seis meses, totalizando $2.880 \mathrm{~km}$ percorridos. Concentramos as amostragens no período mais quente do ano, de setembro de 2014 a fevereiro de 2015, quando se espera maior atividade de aves e répteis, além da presença de grande parte das aves migratórias (GIBBONS; SEMLITSCH, 1987; SICK, 1997). Amostragens neste período permitem estimativas de riqueza mais robustas, mesmo que não comtemplem toda a sazonalidade climática.

Para cada indivíduo encontrado atropelado foi realizado o registro do local (georeferenciado), data, período do dia e registro fotográfico. Foram consideradas todas as carcaças visualizadas na pista e acostamento. Após o registro, as carcaças foram removidas para fora da pista. Foram registrados indivíduos atropelados de aves, mamíferos e répteis. A identificação foi feita no menor nível taxonômico possível. Para análises foram utilizados indivíduos identificados em nível de espécie ou gênero. A identificação dos animais seguiu literatura especializada, livros guias e consulta a especialistas (SICK, 1997;
AZEVEDO; GUIZONI JR., 2008; REIS et al., 2006; WEBER et al., 2013; HARTMANN et al., 2009)

\section{Análise dos dados}

Com a frequência das amostragens foi possível estimar a taxa de atropelamentos (atropelamentos $/ \mathrm{km} / \mathrm{dia}$ ). Para calcular a taxa diária de atropelamentos foi dividido o número total de indivíduos encontrados atropelados pelo comprimento do trecho amostral $(24 \mathrm{Km})$ e pelo número de dias do período amostral (60 dias). A curva de acumulação de espécies foi calculada a partir de 500 adições aleatórias das amostras, utilizando o programa EstimateS 9.0 (COLWELL, 2012). Adicionalmente, estimouse a riqueza de espécies com base no uso do estimador de riqueza Jacknife 1.

Para determinar as espécies mais atropeladas foram selecionadas aquelas que apresentarem, em média, um ou mais indivíduos atropelados por mês, ou seja, $\mathrm{n} \geq 06$. Para verificar se houve diferenças no número de atropelamentos entre os trechos amostrados utilizou-se o teste de qui quadrado $\left(\chi^{2}\right)$.

As espécies foram classificadas segundo o índice de Constância de Ocorrência (DAJOZ, 2005), através da fórmula:

$$
\mathrm{C}=(\mathrm{P} / \mathrm{Q}) \times 100
$$

onde, $\mathrm{C}$ = constância de ocorrência da espécie; $\mathrm{P}$ = número de amostragens em que a espécie ocorreu e $\mathrm{Q}=$ número total de amostragens. A partir da frequência de ocorrência (C) de cada espécie foram obtidas as seguintes categorias: espécie constante $(C>50 \%)$, espécie acessória $(25 \%>C<50 \%)$ e espécie rara $(C<25 \%)$. Para o cálculo do Índice de Constância de Ocorrência foi considerado cada bloco de amostragem (5 dias) como uma unidade amostral (DAJOZ, 2005).

A partir das espécies identificadas (total e por grupo) e da presença delas em cada trecho amostrado, procedeu-se uma análise de similaridade, através da 
seguinte fórmula:

$$
S=(E E \times 100) / T
$$

onde, $\mathrm{S}=$ Similaridade, $\mathrm{EE}=$ total de espécies exclusivas e $\mathrm{T}=$ Total de espécies.

\section{Resultados}

Foram encontrados atropelados 209 indivíduos pertencentes a 45 espécies de três grupos: aves, mamíferos e répteis. A maior abundância e riqueza foram de aves, 120 indivíduos de 30 espécies $(66,7 \%)$, seguidas pelos mamíferos, 52 indivíduos de oito espécies $(17,8 \%)$ e répteis, com 37 indivíduos de sete espécies (15,5\%; Tabela 1). A porcentagem de indivíduos não identificados representou $34,92 \%(n=73)$. A maior parte dos indivíduos não identificados pertence ao grupo das aves $(n=65 ; 31,1 \%)$. Uma espécie de mamífero consta na lista da fauna ameaçada de extinção do Estado do Rio Grande do Sul (RIO GRANDE DO SUL, 2014) e na Lista Vermelha da União Internacional de Conservação da Natureza (IUCN, 2015): Leopardus tigrinus (gato-do-mato-pequeno), em ambas na categoria "vulnerável".

A taxa de atropelamento foi de 0,14 ind. $/ \mathrm{km} / \mathrm{dia}$ considerando os três grupos taxonômicos. Quando analisada por grupos, a maior taxa de atropelamento foi das aves $(0,08$ ind. $/ \mathrm{km} /$ dia $)$, seguido de mamíferos $(0,03$ ind. $/ \mathrm{km} / \mathrm{dia})$ e répteis $(0,02 \mathrm{ind} . / \mathrm{km} / \mathrm{dia})$. A riqueza estimada (Jackknife 1) considerando todos os grupos foi de 67 espécies $( \pm 4,06)$. Para aves a riqueza estimada foi de 46,5 espécies $( \pm 3,71)$; 11,67 espécies para mamíferos $( \pm 1,56)$ e 8,83 espécies para répteis $( \pm 1,24 ;)$.

Tabela 1 - Classe, espécie, número de indivíduos por trecho amostrado, número total de indivíduos, porcentagem total e por grupo, Constância de Ocorrência (C) e classificação segundo o índice de Constância de Ocorrência (C = constante, A = acessória, $\mathrm{R}=$ rara), de aves, mamíferos e répteis encontrados atropelados em dois trechos de $12 \mathrm{~km}$ das rodovias: RS331(T1), entre os municípios de Erechim e Gaurama e RS- 420 (T2), entre os municípios de Erechim e Aratiba, no Estado do Rio Grande do Sul, de setembro de 2014 a fevereiro de 2015. Em negrito as espécies mais atropeladas

\begin{tabular}{lccccccc}
\hline \multicolumn{1}{c}{ Classe/espécie } & $\mathrm{T} 1$ & $\mathrm{~T} 2$ & Total & \% Total & $\begin{array}{c}\text { \% no } \\
\text { grupo }\end{array}$ & $\mathrm{C}$ & Classificação \\
\hline AVES & 61 & 59 & 120 & 57,42 & & & \\
\hline Athene cunicularia & 2 & 1 & 3 & 1,43 & 2,50 & 25,00 & $\mathrm{~A}$ \\
Columbina picui & 2 & 1 & 3 & 1,43 & 2,50 & 16,66 & $\mathrm{R}$ \\
Columbina talpacoti & 1 & - & 1 & 0,48 & 0,83 & 8,33 & $\mathrm{R}$ \\
Crotophaga ani & 1 & 3 & 4 & 1,91 & 3,33 & 25,00 & $\mathrm{~A}$ \\
Cyanolaxia brissonii & - & 1 & 1 & 0,48 & 0,83 & 8,33 & $\mathrm{R}$ \\
Euphonia chlorotica & - & 1 & 1 & 0,48 & 0,83 & 8,33 & $\mathrm{R}$ \\
Furnarius rufus & - & 2 & 2 & 0,96 & 1,66 & 16,66 & $\mathrm{R}$ \\
Guira guira & 1 & 2 & 3 & 1,43 & 2,50 & 25,00 & $\mathrm{~A}$ \\
Lanio culcullatus & 1 & 1 & 1 & 0,96 & 1,66 & 16,66 & $\mathrm{R}$ \\
Megascops sp. & 4 & - & 4 & 1,91 & 3,33 & 25,00 & $\mathrm{~A}$ \\
Molothrus bonariensis & - & 1 & 1 & 0,48 & 0,83 & 8,33 & $\mathrm{R}$ \\
Myiophobus fasciatus & 1 & - & 1 & 0,48 & 0,83 & 8,33 & $\mathrm{R}$ \\
Pardirallus nigricans & - & 1 & 1 & 0,48 & 0,83 & 8,33 & $\mathrm{R}$ \\
Pitangus sulphuratus & - & 1 & 1 & 0,48 & 0,83 & 8,33 & $\mathrm{R}$ \\
Poospiza nigrorufa & 1 & - & 1 & 0,48 & 0,83 & 8,33 & $\mathrm{R}$ \\
Ramphastos dicolorus & - & 1 & 1 & 0,48 & 0,83 & 8,33 & $\mathrm{R}$ \\
Setophaga pitiayumi & 1 & - & 1 & 0,48 & 0,83 & 8,33 & $\mathrm{R}$ \\
Sicalis flaveola & 1 & 1 & 2 & 0,96 & 1,66 & 16,66 & $\mathrm{R}$ \\
Sporagra magellanica & - & 1 & 1 & 0,48 & 0,83 & 8,33 & $\mathrm{R}$ \\
& & & & & & & continua..
\end{tabular}


Tabela 1 - Classe, espécie... continuação

\begin{tabular}{|c|c|c|c|c|c|c|c|}
\hline Classe/espécie & $\mathrm{T} 1$ & $\mathrm{~T} 2$ & Total & $\%$ Total & $\begin{array}{l}\% \text { no } \\
\text { grupo }\end{array}$ & $\mathrm{C}$ & Classificação \\
\hline AVES & $\cdots$ & -- & 1 & $--\cdot$ & & & \\
\hline Sporophila caerulescens & 1 & - & 1 & 0,48 & 0,83 & 8,33 & $\mathrm{R}$ \\
\hline Sporophila sp. & 1 & - & 1 & 0,48 & 0,83 & 8,33 & $\mathrm{R}$ \\
\hline Tangara preciosa & 1 & - & 1 & 0,48 & 0,83 & 8,33 & $\mathrm{R}$ \\
\hline Tangara sayaca & - & 2 & 2 & 0,96 & 1,66 & 8,33 & $\mathrm{R}$ \\
\hline Thamnophilus ruficapillus & 1 & - & 1 & 0,48 & 0,83 & 8,33 & $\mathrm{R}$ \\
\hline Troglodytes musculus & 1 & 1 & 2 & 0,96 & 1,66 & 16,66 & $\mathrm{R}$ \\
\hline Turdus rufiventris & 1 & 1 & 2 & 0,96 & 1,66 & 16,66 & $\mathrm{R}$ \\
\hline Tyrannus melancholicus & 1 & - & 1 & 0,48 & 0,83 & 8,33 & $\mathrm{R}$ \\
\hline Vanellus chilensis & 2 & - & 2 & 0,96 & 1,66 & 16,66 & $\mathrm{R}$ \\
\hline Zenaida auriculata & 1 & - & 1 & 0,48 & 0,83 & 8,33 & $\mathrm{R}$ \\
\hline Zonotrichia capensis & 4 & 3 & 7 & 3,35 & 5,83 & 58,33 & $\mathbf{C}$ \\
\hline Não identificados & 31 & 34 & 65 & 31,10 & 54,16 & & \\
\hline MAMÍFEROS & 26 & 26 & 52 & 24,88 & & & \\
\hline Cavia aperea & 4 & 2 & 6 & 2,87 & 11,54 & 33,33 & $\mathbf{A}$ \\
\hline Cerdocyon thous & 3 & 5 & 8 & 3,83 & 15,38 & 50,00 & $\mathbf{A}$ \\
\hline Dasypus novemcinctus & 3 & 1 & 4 & 1,91 & 7,69 & 25,00 & $\mathrm{~A}$ \\
\hline Didelphis albiventris & 8 & 14 & 22 & 10,52 & 42,31 & 100,00 & $\mathbf{C}$ \\
\hline Galictis cuja & 1 & - & 1 & 0,48 & 1,92 & 8,33 & $\mathrm{R}$ \\
\hline Leopardus tigrinus & - & 1 & 1 & 0,48 & 1,92 & 8,33 & $\mathrm{R}$ \\
\hline Lepus europaeus & 1 & - & 1 & 0,48 & 1,92 & 8,33 & $\mathrm{R}$ \\
\hline Myocastor coypus & - & 1 & 1 & 0,48 & 1,92 & 8,33 & $\mathrm{R}$ \\
\hline Não identificados & 6 & 2 & 8 & 3,83 & 15,38 & & \\
\hline RÉPTEIS & 19 & 18 & 37 & 17,7 & & & \\
\hline Atractus taeniatus & 2 & 1 & 3 & 1,43 & 8,11 & 25,00 & $\mathrm{~A}$ \\
\hline Bothrops jararaca & - & 3 & 3 & 1,43 & 8,11 & 25,00 & $\mathrm{~A}$ \\
\hline Micrurus altirostris & 2 & - & 2 & 0,96 & 5,41 & 16,66 & $\mathrm{R}$ \\
\hline Oxyrhopus rhombifer & 1 & - & 1 & 0,48 & 2,70 & 8,33 & $\mathrm{R}$ \\
\hline Philodryas olfersii & - & 1 & 1 & 0,48 & 2,70 & 8,33 & $\mathrm{R}$ \\
\hline Salvator merianae & 11 & 13 & 24 & 11,48 & 64,86 & 58,33 & $\mathbf{C}$ \\
\hline Thamnodynastes strigatus & 3 & - & 3 & 1,43 & 8,11 & 16,66 & $\mathrm{R}$ \\
\hline TOTAL & 106 & 103 & 209 & 100 & & & \\
\hline
\end{tabular}


A curva com maior tendência à estabilização no número de espécies foi para o grupo dos répteis (Figura 2D). Para os três grupos juntos, aves e mamíferos não houve tendência de estabilização (Figura 2 A, B e C).

As espécies com mais registros de atropelamentos foram: Salvator merianae (Teiú; $\mathrm{n}=24$ ), Diadelfos albiventris (Gambá-de-orelha-branca; $\mathrm{n}=22$ ), Cerdocyon thous (Graxaim-do-mato; $\mathrm{n}=8$ ), Zonotrichia capensis (Tico-tico; $\mathrm{n}=7$ ) e Cavia aperea (Preá; $\mathrm{n}=6$ ). As cinco espécies mais atropeladas representaram $32 \%$ dos registros totais $(\mathrm{n}=67$; Tabela 1$)$

Dentre aves, a espécie mais atropelada foi Z. Capensis, com 3,35\% dos registros totais de atropelamentos e 5,33\% dos registros de atropelamentos no grupo das aves. Zonotrichia capensis foi classificada como Constante sendo encontrada em sete das doze amostragens realizadas. Três espécies de mamíferos estiveram entre as mais atropeladas, D. albiventris, C. thous, e C. aperea, que representaram $17,22 \%$ do total de registros e $69,23 \%$ dos registros para mamíferos. Didelphis albiventris foi classificada como Constante e foi a única espécie que apareceu em todas as amostragens realizadas. Cerdocyon thous e C. aperea foram classificadas como espécies Acessórias e apareceram em seis e quatro amostragens respectivamente. $\mathrm{O}$ atropelamento de S. merianae representou $11,48 \%$ dos registros totais e $64,86 \%$ dos registros de répteis. Salvator merianae foi classificada como Constante e foi registrada em sete amostragens.

No trecho T1 foram encontrados 106 indivíduos atropelados, sendo 61 aves, 26 mamíferos e 19 répteis. No trecho T2 foram encontrados 103 animais atropelados, sendo 59 de aves, 26 de mamíferos e 18 de répteis (Figura 3).

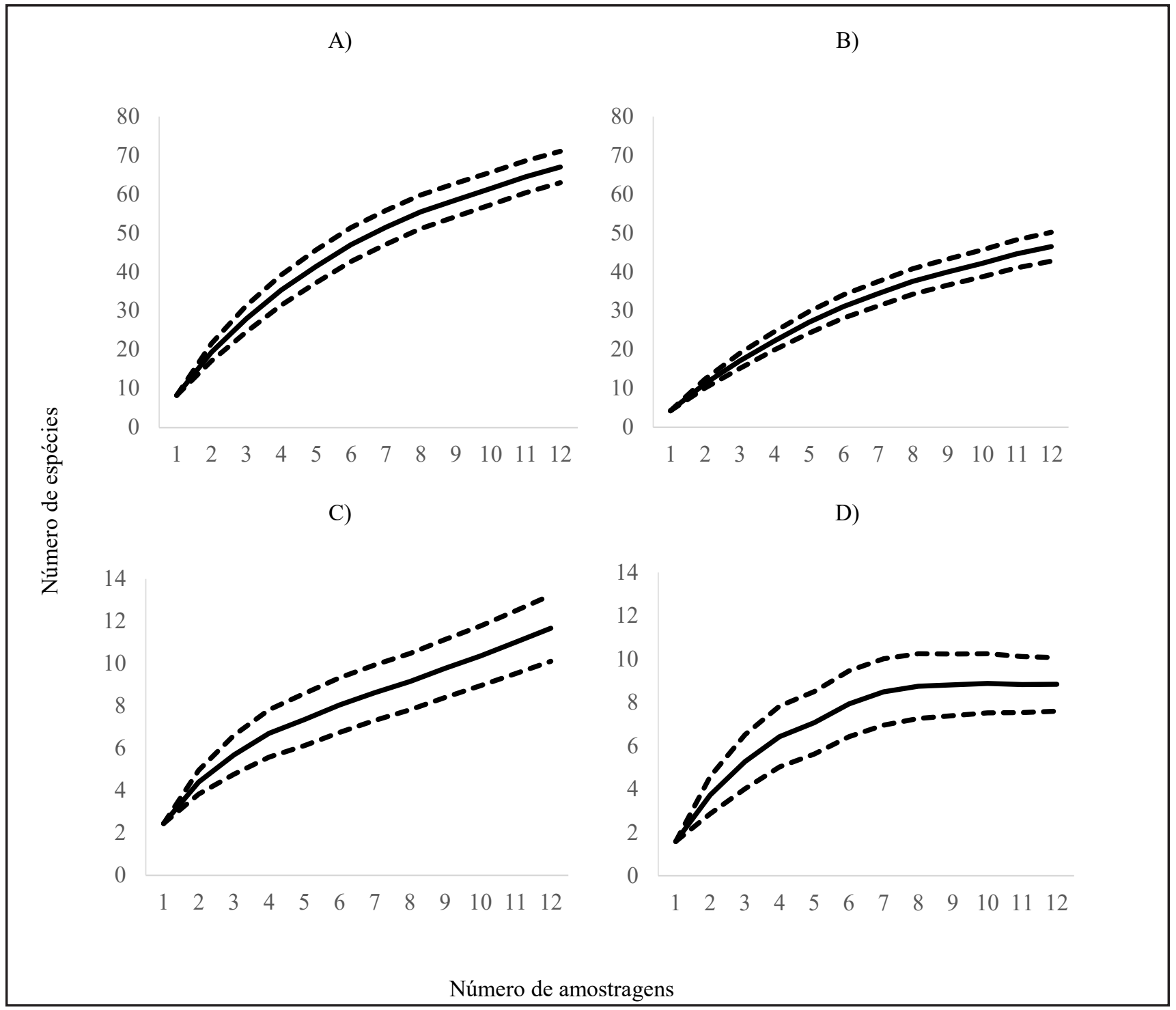

Figura 2: Curvas de acumulação de espécies de aves, mamíferos e répteis encontradas atropeladas em dois trechos de 12 km: RS-331, entre os municípios de Erechim e Gaurama e RS-420, entre os municípios de Erechim e Aratiba, de setembro de 2014 a fevereiro de 2015. A) Para todos os grupos; B) Aves; C) Mamíferos; D) Répteis. Linha contínua: Riqueza estimada. Linhas tracejadas: Desvio padrão. 


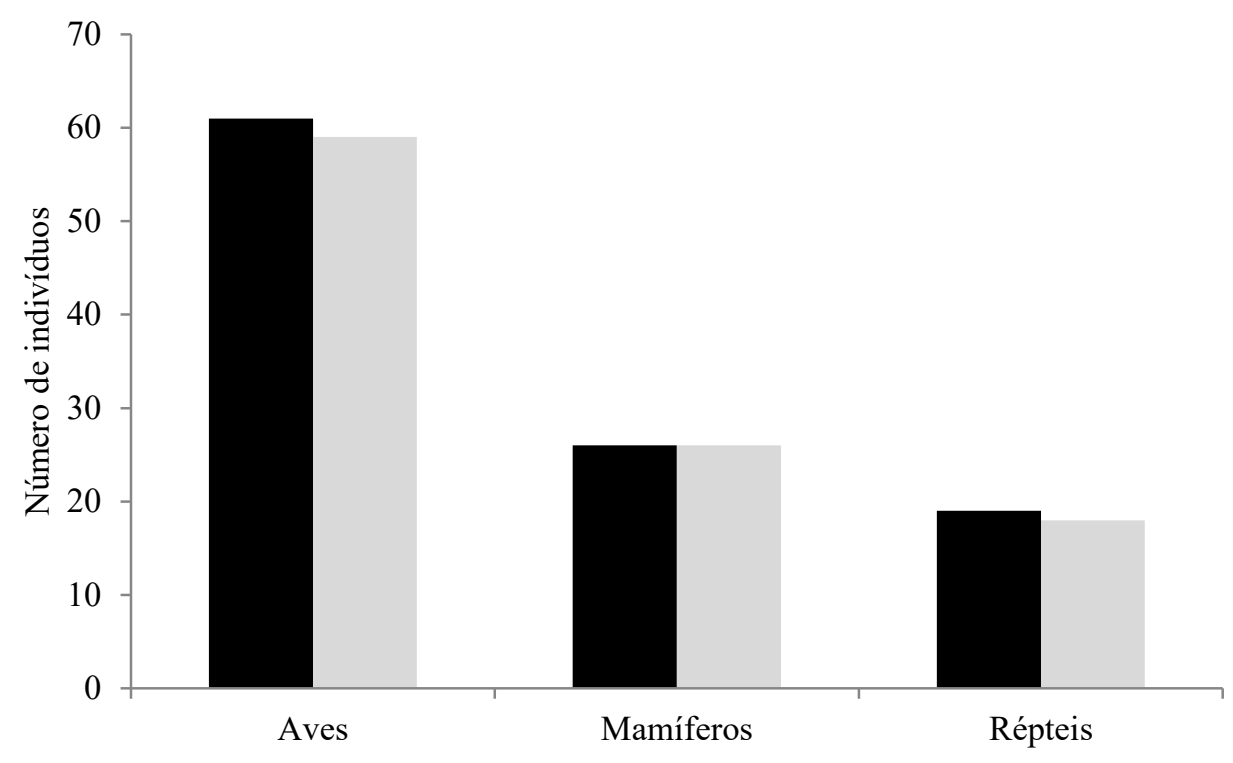

Figura 3 - Número de indivíduos de aves, mamíferos e répteis encontrados atropelados em dois trechos de 12 km: RS-331 (T1, coluna preta), entre os municípios de Erechim e Gaurama e RS-420 (T2, coluna cinza), entre os municípios de Erechim e Aratiba, de setembro de 2014 a fevereiro de 2015

Tabela 2 - Número de espécies encontradas atropeladas em dois trechos de 12 km das rodovias: RS-331 (T1), entre os municípios de Erechim e Gaurama e RS-420 (T2), entre os municípios de Erechim e Aratiba, no Estado do Rio Grande do Sul, de setembro de 2014 a fevereiro de 2015. Total de espécies por grupo; Similaridade (S) entre os trechos e espécies exclusivas do trecho T1 e T2 (ET1 e ET2)

\begin{tabular}{lcccccc}
\hline Táxon & T1 & T2 & Total & S & ET1 & ET2 \\
\hline Aves & 21 & 18 & 30 & $9(30,0 \%)$ & 12 & 9 \\
Mamíferos & 6 & 6 & 8 & $4(50,0 \%)$ & 2 & 2 \\
Répteis & 5 & 4 & 7 & $2(28,5 \%)$ & 3 & 2 \\
\hline Total & 32 & 28 & 45 & $15(33,3 \%)$ & 17 & 13 \\
\hline
\end{tabular}

Não houve diferença no total de animais atropelados entre os dois trechos $\left(\chi^{2}=0,02 ; G L=1 ; p=0,88\right)$. O grupo das aves foi o mais atropelado em ambos os trechos e também não houve diferença entre o número de indivíduos de aves atropelados entre os trechos $\left(\chi^{2}=0,02 ; \mathrm{GL}=\right.$ $1 ; p=0,89)$. Da mesma forma para mamíferos $\left(\chi^{2}=0,00\right.$; $\mathrm{GL}=1 ; \mathrm{p}=1)$ e répteis $\left(\chi^{2}=0,01 ; \mathrm{GL}=1 ; \mathrm{p}=0,90\right)$.

Das 45 espécies identificadas, apenas 15 foram encontradas nos dois trechos (similaridade $=33,3 \%$; Tabela 2). Para aves foram nove espécies (similaridade $=30,0 \%$ ), para mamíferos foram quatro (similaridade $=50 \%$ ) e para répteis duas (similaridade $=28,5 \%$ ). Foram encontradas 17 espécies atropeladas exclusivamente no trecho T1, sendo 12 espécies de aves, duas de mamíferos e três de répteis. No trecho T2, foram encontradas 13 espécies exclusivas, sendo nove de aves, duas de mamíferos e duas de répteis.

Os meses de novembro e dezembro de 2014 e janeiro de 2015 foram os meses com maior número de animais atropelados. O grupo das aves foi afetado em todos os meses, com maior registro em novembro $(n=31)$. Os mamíferos também apresentaram indivíduos atropelados em todos os meses, sendo o mês de dezembro o mês com maior número de registros $(\mathrm{n}=15)$. Atropelamentos de répteis foram registrados a partir do mês de novembro, sendo o maior número de indivíduos encontrados atropelados no mês de janeiro $(n=13)$.

\section{Discussão}

Embora 45 espécies tenham sido identificadas, as maiores taxas de atropelamento foram concentradas em poucas espécies. As espécies que foram mais atropeladas são abundantes na região e com ampla distribuição geográfica (SILVA, 1984; SICK, 1997). Situação semelhante foi descrita em outros estudos, indicando que as espécies mais atropeladas são geralmente generalistas, localmente abundantes, com grande capacidade de deslocamento e/ou aquelas que são atraídas pelos recursos ou carac- 
terísticas ambientais favoráveis nas estradas (FORMAN et al., 2003; COELHO et al., 2008; GRILO et al., 2009; HARTMANN et al., 2011; HARTMANN et al., 2012).

As curvas de acumulação de espécies indicam que novos registros podem ser feitos com mais tempo de amostragem. Ou seja, que o número de espécies potencialmente afetadas por atropelamentos na região é maior que a registrada neste estudo, pois todos os grupos apresentaram novos registros efetuados até o final da amostragem. Provavelmente, são animais que não são atropelados com frequência, pelo pouco contato que tem com as rodovias, maior atividade em períodos não amostrados, ou por possuem maior habilidade em atravessá-la (CUNHA et al., 2015).

Determinadas espécies de aves parecem altamente susceptíveis a atropelamentos (RODRIGUES et al., 2002; PRADA, 2004; BAGATINI, 2006). Clevenger et al. (2003), citam que o voo e o forrageamento na estrada são fatores que aumentam a vulnerabilidade das aves ao atropelamento. O pequeno peso corpóreo dos indivíduos e a constituição delicada de muitas espécies de aves faz com que também sejam atingidas pelo forte deslocamento de ar provocado pela passagem dos veículos em alta velocidade (PRADA, 2004), além da colisão com veículos. A espécie de ave com mais registros de atropelamentos no estudo (Zonotrichia capensis) tem alta capacidade de deslocamento entre os fragmentos vegetais e pode buscar alimento no solo (SICK, 1997), inclusive em rodovias, o que pode potencializar os atropelamentos.

Os mamíferos estão entre os mais frequentemente encontrados atropelados. Muitos estudos têm atenção especial a esse grupo (VIEIRA, 1996; SCOSS, 2002; PEREIRA et al., 2006; BUENO; ALMEIDA, 2010; HEGEL et al., 2012) ou apresentam como o grupo mais atropelado entre os vertebrados (OLIVEIRA; SILVA, 2012; SANTANA, 2012). Para mamíferos, características como o tamanho corporal e a dieta podem influenciar quais espécies são mais suscetíveis ao atropelamento (CÁCERES et al., 2010). Animais maiores precisam de maiores áreas de vida além de se deslocarem mais pela paisagem, aumentando a probabilidade de encontrar uma estrada e atravessá-la (FORMAN; ALEXANDER, 1998). A dieta pode influenciar, pois algumas espécies de herbívoros apresentam maiores densidades populacionais que os carnívoros ou onívoros e são atraídas pelas gramíneas existentes ao longo das bordas das estradas (BENNET, 1991).

O mamífero com maior número de registros de atropelamento foi $D$. albiventris. Isso pode estar relacionado ao fato dessa espécie ser oportunista em relação ao uso do ambiente e abundante no sul do Brasil (SILVA, 1984). Espécies de Didelphis estiveram entre as mais atropeladas na região central (RODRIGUES et al., 2002) e sul do Brasil (ROSA; MAUHS, 2004; CHEREM et al., 2007). Cerdocyon thous também está entre as espécies mais atropeladas em outros estudos (VIEIRA, 1996; CÂNDIDO-JR. et al., 2002; RODRIGUES et al., 2002; PINOWSKI, 2005). Essa espécie é onívora, oportunista e com grande mobilidade, que pode utilizar a estrada para forrageio e deslocamento, aumentado a possibilidade de atropelamento (CHEIDA et al., 2006; HENGEMÜHLE; CADEMARTORI, 2008). Cavia aperea tem como habitat os estratos herbáceos e pode ocupar as gramíneas localizadas no entorno da maioria das rodovias (WEBER et al., 2013). É abundante na região e parece ocupar as margens da rodovia para forrageamento (obs. pes.).

As taxas de atropelamento de répteis podem variar em função das estações do ano e paisagem no entorno da rodovia (HARTMANN et al., 2011; HARTMANN et al., 2012; RODRIGUES et al., 2002; ROSA; MAUHS, 2004; SILVA et al., 2007). Neste estudo, os répteis começaram a ser encontrados em novembro, dois meses depois do início das amostragens, indicando que a estação do ano influenciou no atropelamento. Apesar da maioria dos répteis terem uma movimentação menor que as aves e os mamíferos, outras características podem influenciar na susceptibilidade aos atropelamentos, como a baixa velocidade no deslocamento e respostas comportamentais à luz, como imobilidade (PRADA, 2004).

A abundância de $S$. merianae, assim como suas características ecológicas podem potencializar o atropelamento. Rosa; Mauhs (2004) registraram a espécie com 32,8\%, porém não foi registrado durante as estações de outono e inverno. Neste estudo, $S$. merianae foi registrado a partir de novembro 2014, durante o período mais quente do ano, quando as maiorias das espécies de répteis estão mais ativas (GIBBONS; SEMLITSCH, 1987). Aparentemente, a variação na temperatura ao longo do ano influencia na movimentação da espécie, o que poderia aumentar a sua exposição ao atropelamento.

As espécies com maior número de atropelamentos coincidem em grande parte com as espécies classificadas como Constantes. Isto indica que a maior incidência de atropelamentos está associada, pelo menos em parte, às espécies com exposição continuada à rodovia, ou seja, temporalmente não concentrada. Essa continuidade na mortalidade nas estradas pode ter influência no tamanho das populações das espécies frequentemente atingidas, pois se não há um período claro de recuperação do número de indivíduos, pode ocorrer a diminuição lenta, mas constante na abundância das espécies (CLEVENGER et al., 2003).

Neste estudo, três espécies classificadas como constantes, e destas, somente D. albiventris apareceu em todas as amostragens. Fatores como provável abundância no entorno, hábitos alimentares generalistas e a capacidade de ocupar habitats antropizados podem fazer com que esta espécie esteja constantemente sujeita aos atropelamentos (ROSA; MAUHS, 2004). Salvator merianae também foi classificado como constante, possivelmente em função das amostragens terem sido realizadas fora das estações frias, quando aparentemente $S$. merianae reduz sua atividade (ROSA; MAUHS, 2004). Zonotrichia capensis também foi classificada como constante e isso pode estar 
associado aos seus constantes deslocamentos entre os fragmentos florestais, pela sua adaptação em habitats antropizados e pelo seu hábito de buscar alimento nas rodovias (SICK, 1997).

Taxas de atropelamento tendem a variar em função do grupo taxonômico avaliado, bioma amostrado, frequência nas amostragens e comprimento do trecho amostrado (ver HANGEMÜHLE; CADEMARTORI, 2008; FISCHER, 1997; SILVEIRA, 1999; PRADA, 2004; MANTOVANI, 2001; HARTMANN et al., 2012; CUNHA et al., 2015). Deve-se levar em consideração que o número de animais atropelados, deve estar subestimado, pois há limitações inerentes ao método, remoção de carcaças por animais carniceiros, além de animais feridos, que podem se afastar para longe da rodovia, influenciando na taxa de mortalidade (VIEIRA, 1996; RODRIGUES et al., 2002). Antworth et al. (2005), em um estudo experimental verificaram que $60 \%$ das carcaças de aves e $97 \%$ das de répteis foram removidas em até 36 horas após a colocação das mesmas na rodovia. No entanto, o uso de taxas permite determinar o corte inferior do número de atropelamentos. A taxa estimada de atropelamentos neste estudo representa que nos períodos mais quentes do ano podem ser encontrados ao menos 50 animais atropelados por quilômetro nas rodovias avaliadas.

Dentre os indivíduos encontrados atropelados, uma espécie está ameaçada de extinção, Leopardus tigrinus. De acordo com Oliveira; Cassaro (1999), todas as espécies de felinos silvestres encontrados em território brasileiro estão ameaçadas de extinção devido à destruição do meio ambiente. As rodovias estão atualmente entre os principais meios para o declínio desse grupo (CHEREM et al., 2007; CAMARGO et al., 2011; HEGEL et al., 2012; OLIVEIRA; SILVA, 2012).

A presença de espécies exclusivas para cada área do estudo parece ser influenciada pelas diferenças nas características ambientais do entorno das rodovias. No trecho $\mathrm{T} 1$ a paisagem do entorno na rodovia apresenta grandes áreas antropizadas (agricultura e pecuária). No trecho T2 a paisagem tem relevo de ondulações acentuadas e predomínio de vegetação florestal na encosta dos morros, intercalada com pequenas áreas agrícolas nas áreas planas. Esta condição mais preservada parece favorecer espécies mais exigentes em relação ao habitat. A similaridade média a baixa e as diferenças na diversidade entre os dois trechos indicam possível influência da paisagem do entorno da rodovia na diversidade de fauna atropelada. Rodovias que cortam áreas mais degradadas parecem gerar impactos sobre animais de hábitos generalistas, com alta capacidade de ocupar áreas antrópicas e aparentemente com populações grandes. Por outro lado, rodovias que cortam áreas preservadas, ou menos degradadas, tendem a gerar impactos sobre espécies mais exigentes ao uso de habitat, com áreas de vida mais restritas e possivelmente populações menores. Comumente são estas as espécies que mais demandam atenção para conservação.
A redução dos atropelamentos de animais silvestres depende de ações educativas e implantação de medidas mitigadoras. Pelo fato de algumas medidas terem eficácia incerta e serem relativamente dispendiosas (TROMBULAK; FRISSEL, 2000) é necessário conhecer bem os fatores envolvidos nas ocorrências de cada região. Muitas vezes as medidas usadas não têm correlação direta com os resultados, sendo necessárias avaliações baseadas na eficácia das medidas para os grupos alvo (FORMAN et al., 2003). Algumas medidas mitigadoras apresentam um sucesso parcial ou foram criadas para uso de uma determinada espécie e são usadas por outras (TROMBULAK; FRISSEL, 2000; CLEVENGER et al., 2003). Neste sentido, a identificação das espécies mais frequentemente atropeladas em cada região e/ou paisagem e dos padrões ecológicos envolvidos se mostra fundamental para definir a necessidade de implantação e quais medidas mitigadoras são mais adequadas.

\section{Agradecimentos}

Agradecemos a Dra. Noeli Zanella e Dra. Elaine Lucas Gonsales pelas sugestões nas versões prévias do artigo. Agradecemos também a Guilhermo Romero pelo auxílio na elaboração do mapa da localização da área de estudo. Alexandre Folador, Cassiane Kolcenti, Catiusa Rosin, Daiani Brandler, Inês Rezendes, Renata Treméa, Heloísa Dalla Rosa e Liane Ruszczyk que fornecerem valioso auxílio nos trabalhos de campo.

\section{Referências}

AB'SABER, A.; MARIGO, L. Ecossistemas do Brasil. São Paulo: Metalivros, 2006. 300p.

ANDREWS, K.M.; GIBBONS, J.W. How do highways influence snake movement? Behavioral responses to roads and vehicles. Copeia, v. 2005, n. 4, p. 772-782, 2005.

ANTWORTH, R.L.; PIKE, D.A.; STEVENS, E.E. Hit and run: effects of scavenging on estimates of roadkiled vertebrates. Southeastern Naturalist, v. 4, n. 4, p. 647656, 2005.

AZEVEDO, M.A.G.; GHIZONI-JR, I.R. Aves. In: CHEREM, J.J.; KAMMERS, M. (Orgs) A fauna das áreas de influência da Usina Hidrelétrica Quebra-Queixo. Erechim: Habilis, 2008. p. 131-150.

BAGATINI, T. Evolução dos índices de atropelamento de vertebrados silvestres nas rodovias do entorno da Estação Ecológica de Águas Emendadas, DF Brasil, e eficácia de medidas mitigadoras. Dissertação (Mestrado em Ecologia) - Universidade de Brasília, Brasília, 2006. 
BAGER, A.; LUCAS, P.S.; BOURSCHEIT, A.; KUCZACH, A.; MAIA, B. Os caminhos da conservação da biodiversidade brasileira frente aos impactos da infraestrutura. Biodiversidade Brasileira, v. 6, n. 1, p.75-86, 2016.

BAGER, A.; ROSA, C.A. Impacto da rodovia BR-392 sobre comunidades de aves no extremo sul do Brasil. Revista Brasileira de Ornitologia, v. 20, n. 1, p. 30-39, 2012.

BENÍTZ-LÓPEZ, A.; ALKEMADE, R.; VERWEIJ, P.A. The impacts of roads and other infrastructure on mammal and bird populations: a meta analysis. Biological Conservation, v. 143, p. 1307-1316, 2010.

BENNET, A.F. Roads, roadsides and wildlife conservation: a review. In: SAUNDERS, D.A.; HOBBS, R. J. (Eds) Nature Conservation 2: The Role of Corridors. Chipping Norton: Beatty and Sons, 1991.

BUENO, C.; ALMEIDA, P.A.L. Sazonalidade de atropelamentos e os padrões de movimentos em mamíferos na BR - 040 (Rio de Janeiro - Juiz de Fora). Revista Brasileira de Zoociências, v. 12, n. 3, p. 219-226, 2010.

CÁCERES, N.C.; HANNIBAL, W.; FREITAS, D.R.; SILVA, E.L.; ROMAN, C.; CASELLA, J. Mammal occurrence and roadkill in two adjacent ecoregions (Atlantic Forest and Cerrado) in south-western Brazil. Revista Brasileira de Zoologia, v. 27, p. 709-717, 2010.

CAMARGO, B.M.; MAZIM, F.D.; GARCIAS, F.M.; PETRUZZI, V.M. Variação sazonal e espacial de vertebrados silvestres atropelados em três rodovias do Bioma Pampa, Sul do Brasil. In: Road Ecology Brazil, Lavras, MG. Anais... Lavras, 2011.

CÂNDIDO-JR, J.F.; MARGARIDO, V.P.; PEGORARO, J.L.; D 'AMIGO, A.R.; MADEIRA, W.D.; CASALE, V.C.; ANDRADE, L. Animais atropelados na rodovia que margeia o Parque Nacional do Iguaçu, Paraná, Brasil, e seu aproveitamento para estudos da biologia da conservação. In: III Congresso Brasileiro de Unidades de Conservação, Fortaleza, CE. Anais... Fortaleza, 2002.

CHEIDA, C.C.; NARANO-COSTA, E.; FUSCO-COSTA, R.; ROCHA-MENDES, F.; QUADROS, J. 2006. Mamíferos do Brasil. In: REIS, N.R.; RERACCHI, L.A.; PEDRO, A.W.; LIMA, P.I. (Eds) Ordem Carnívora. Londrina: Edifurb, 2006, p.231-275.

CHEREM, J.J.; KAMMERS, M.; GHIZONI-JR I. R.; MARTINS, A. Mamíferos de médio e grande porte atropelados em rodovias do Estado de Santa Catarina, Sul do Brasil. Biotemas, v. 20, n. 2, p. 81-96, 2007.

CLEVENGER, A.P.; CHRUSZCZ, B.; GUNSON, K.E. Spatial patterns and factors influencing small vertebrate fauna roadkill aggregations. Biological Conservation, v. 109, p. 15-26, 2003.
COELHO, I.P.; KINDEL, A.; COELHO, A.V.P. Roadkills of vertebrate species on two highways through the Atlantic Forest Biosphere Reserve, southern Brazil. European Journal of Wildlife Research, v. 54, p. 689-699, 2008.

COLWELL, R.K. EstimateS 9.0: Interpolating, extrapolating, and comparing incidence-based species accumulation curves. 2004. Disponível em: http://viceroy.eeb.uconn.edu/ estimates/EstimateSPages/EstSUsersGuide/References/ ColwellMaoAndChang2004Sp.pdf. Acesso em: 03 março de 2015.

CUNHA, G.G.; HARTMANN, M.T.; HARTMANN, P.A. Atropelamentos de vertebrados em uma área de Pampa no sul do Brasil. Ambiência, v. 11, n. 2, p. 307-320, 2015.

DAJOZ, R. Princípios de Ecologia.7ed. Porto Alegre: Artmed, 2005.

DRAMASTAD, W.E.; OLSON, J.D.; FORMAN, R.T.T. Landscape ecology principles in landscape architecture and land-use planning. Washington: Island Press, 1996.

ERRITOZOE, J.; MAZGAJSKI, T.D.; REJT, L. Bird casualties on european roads a review. Acta Ornithologica, v. 38, n. 2, p.77-93, 2003.

FAHRIG, L.; PEDLAR, J.H.; POPE, S.E.; TAYLOR, P.D.; WEGENER, J.F. Effect of road traffic on amphibian density. Biological Conservation, v. 73, p. 177-182, 1995.

FAHRIG, L.; RYTWINSKI, T. Effects of roads on animal abundance: an empirical review and synthesis. Ecology and Society, v. 14, n. 1, p. 1-19, 2009.

FEPAGRO. Fundação Estadual de Pesquisa Agropecuária. Disponível em: $<\mathrm{http}: / /$ www.fepagro.rs.gov.br/ > . Acesso em: 30 de junho de 2014.

FISCHER, W.A. Efeitos da BR-262 na mortalidade de vertebrados silvestres: sintese naturalística para a conservação da região do Pantanal, MS. Dissertação (Mestrado em Ecologia e Conservação) - Universidade Federal de Mato Grosso do Sul, Campos Grande. 1997.

FORD, A.T.; FAHRIG, L. Diet and body size of North American mammal road mortalities. Transportation Research Part D, v. 12, n. 7, p. 498-505, 2007.

FORMAN, R.T.T.; ALEXANDER, L.E. Roads and their major ecological effects. Annual Review of Ecology, Evolution, and Systematics, v. 29, p. 207-231, 1998.

FORMAN, R.T.T.; SPERLING, D.; BISSONETTE, J.A., CLEVENGER, A.P.; CUTSHALL, C.D.; DALE, V.H.;FAHRIG, L.; FRANCE, R.; GOLDMAN, C.R.; HEANUE, K.; JONES, J.A.;SWANSON, F.J.;TURRENTINE, T.; WINTER, T.C. Road Ecology. Science and Solutions. Washington: Island Press, 2003. 
FORMAN, R.T.T. Road ecology's promise: What's around the bend? Environment, v. 46, n. 3, p. 8-21, 2004.

GIBBS, J.P.; STEEN, D.A. Trends in sex ratios of turtles in the United States: implications of road mortality. Conservation Biology, v. 19, n. 2, p. 552-556, 2005.

GIBBONS J.W.; SEMLITSCH R.D. 1987. Activity patterns., In: SEIGEL R.A.; COLLINS J.T.; NOVAK S.S. (Eds), Snakes: Ecology and Evolutionary Biology. New York: Macmillan, 1987. p. 396-421

GOOSEM, M. Fragmentation impacts caused by roads through rainforest. Current Science, v. 93, n. 11, p. 15871595, 2007.

GRILO, C.; BISSONETTE, A.J.; REIS, M.S. Spatialtemporal patterns in Mediterranean carnivore Road casualties: Consequences for mitigation. Biological Conservation, v. 142, p. 301-313, 2009.

HARTMANN, P.A.; HARTMANN, M.T.; MARTINS, M. Ecology of a snake assemblage in the Atlantic Forest of southeastern Brazil. Papéis Avulsos de Zoologia, v. 49, p. 343-360, 2009.

HARTMANN, P. A.; HARTMANN, M. T.; MARTINS, M. Snake road mortality in a protected area in the Atlantic Forest of southeastern Brazil. South American Journal of Herpetology, v. 6, p. 35-42, 2011.

HARTMANN, P.A.; MAINARDI, L.; REBELATO, M.M.; DELABARY, B.F. Ecologia de estradas no Pampa brasileiro: A perda de répteis por atropelamentos. In: BAGER, A. Ecologia de Estradas: Tendências e Pesquisas. Lavras: UFLA, 2012. p. 153-166.

HEGEL, C.G.Z.; CONSALTER, G.C.; ZANELLA, N. Mamíferos silvestres atropelados na rodovia RS-135, norte do Estado do Rio Grande do Sul. Biotemas, v. 25, n. 2, p. 165-170, 2012.

HENGEMÜHLE, A.; CADEMARTORI, C.V. Levantamento de mortes de vertebrados silvestres devido a atropelamento em um trecho da estrada do mar (RS389). Biodiversidade Pampeana, v. 6, n. 2, p. 4-10, 2008.

HUIJSER, M.P.; BERGERS, P.J.M. The effect of roads and traffic on hedgehog (Erinaceus europaeus) populations. Biological Conservation, v. 95, p. 111-116, 2000.

IBGE. Instituto Brasileiro de Geografia e Estatística. 2010. Disponível em: http://www.ibge.gov.br. Acesso em: 14 dez. 2015.

IUCN. Red list of Threatened Species. 2015. Disponível em: http://www.iucnredlist.org/. Acesso em: 05 jan. 2015.
JAEGER, J.A. G.; BOWMAN, J.; BRENNAN, J.; FAHRIG, L.; BERT, D.; BOUCHARD, J.; CHARBONNEAU, N.; FRANK, K.; GRUBER, B.; VON TOSCHANOWITZ, K.T. Predicting when animal populations are at risk from roads: an interactive model of road voidance behavior. Ecological Modelling, v. 185, p. 329-348, 2005.

LANGEN, T.A.; MACHNIAK, A.; CROWE, E.K. MANGAN, C.; MARKER, D.F.; LIDDLE, N.; RODEN, B. Methodologies for surveying Herpetofauna mortality on rural highways. The Journal of Wildlife Management, v. 71, p. 1361-1368, 2007.

LAURANCE, W.F.; GOOSEM, M.; LAURANCE, S.G.W. Impacts of roads and linear clearings on tropical forests. Trends in Ecology E Evolution, v. 4, n. 12, p. 659-669, 2009.

MANTOVANI, J.L. Telemetria convencional e via satélite na determinação das áreas de vida de três espécies de carnívoros da região nordeste do estado de São Paulo. Tese (Doutorado em Ciências) - Centro de Ciências Biológicas e da Saúde, Universidade Federal de São Carlos, São Carlos. 2001.

OLIVEIRA, T.G.; CASSARO, K. 1999. Guia De Identificação Dos Felinos Brasileiros. São Paulo: Sociedade De Zoológicos do Brasil, 1999.

OLIVEIRA, D.S.; SILVA, V.M. Vertebrados silvestres atropelados na BR 158, RS, Brasil. Biotemas, v. 25, n. 4, p. 229-235, 2012.

PEREIRA, A.P.F.G.; ANDRADE, F.A.G.; FERNANDES, M.E.B. Dois anos de monitoramento dos atropelamentos de mamíferos na rodovia PA- 458, Bragança, Pará. Boletim do Museu Paraense Emílio Goeldi. Ciências Naturais, v. 1, p. 77-83, 2006.

PINOWSKI, J. Roadkills of vertebrates in Venezuela. Revista Brasileira de Zoologia, v. 22, n. 1, p.191-196, 2005.

PRADA, C.S. Atropelamento de vertebrados silvestres em uma região fragmentada do nordeste do estado de São Paulo: quantificação do impacto e análise dos fatores envolvidos. Dissertação (Mestrado em Ecologia e Recursos Naturais) - Centro de Ciências Biológicas e da Saúde, Universidade Federal de São Carlos, São Carlos. 2004.

REIS, N.R.; PERACCHI, A.L.; PEDRO, W.A.; LIMA, I.P. Mamíferos do Brasil. Londrina: UEL, 2006.

RIO GRANDE DO SUL. Decreto $n^{\circ}$ 51797. Lista das espécies da fauna gaúcha ameaçadas de extinção. Diário Oficial do Estado do Rio Grande do Sul, Porto Alegre, 2014.

RODRIGUES, E.; PINHEIRO, J.; PEREIRA, A.P. Estradas e EIA/RIMA no Acre. 2002. Disponível em: http://sites.google. com/site/ecologiaestradas/download. Acesso em: 10 jun. 2015. 
ROSA, A.O.; MAUHS, J. Atropelamentos de animais silvestres na Rodovia RS-40. Caderno de Pesquisa. Série Biologia (UNISC), v. 16, n. 1, p. 35-42, 2004.

SANTANA, G.S. Fatores influentes sobre atropelamentos de vertebrados na região central do Rio Grande do Sul, Brasil. Neotropical Biology and Conservation, v. 7, p. 26-40, 2012.

SCOSS, L.M. Impacto de estradas sobre mamíferos terrestres: O caso do parque estadual do Rio Doce, Minas Gerais. Dissertação de Mestrado, Universidade Federal de Viçosa, 2002.

SICK, H. 1997. Ornitologia brasileira. Rio de Janeiro: Nova Fronteira. 1997, 912p.

SILVA, F. Mamíferos Silvestres - Rio Grande do Sul. Porto Alegre, Fundação Zoobotânica do Rio Grande do Sul. 1984, 246 p.

SILVA, M.O.; OLIVEIRA, I.S.; CARDOSO, M.W.; GRAF, V. Road kills impact over the herpetofauna of Atlantic Forest (PR-340, Antonina, Paraná). Acta Biologica Paranaense, v. 36, n. 1-2, p. 103-112, 2007.

SILVEIRA, L. Ecologia e conservação dos mamíferos carnívoros do Parque Nacional das Emas. Dissertação (Mestrado em Ecologia) - Departamento de Biologia Geral, Universidade Federal de Goiás, 1999.
TROMBULAK, S.C.; FRISSELL, C.A. Review of ecological effects of roads on terrestrial and aquatic communities. Conservation Biology, v. 14, p. 18-30, 2000.

TUMELEIRO, L.K.; KOENEMANN, J.G.; ÁVILA, M.C.N.; PANDOLFO, F.; OLIVEIRA. E.V. Notas sobre mamíferos da região de Uruguaiana: estudo de indivíduos atropelados com informações sobre a dieta e conservação. Biodiversidade Pampeana, v. 4, p. 38-41, 2006.

TURCI, L.C.B.; BERNARDE, P.S. Vertebrados atropelados na Rodovia Estadual 383 em Rondônia, Brasil. Biotemas, v. 22, n. 1, p. 121-127, 2009.

VAN DER REE, R.; GAGNON, J.W.; SMITH, D.J.A. Valuable Tool for Reducing Wildlife-Vehicle Collisions and Funnelling Fauna to Crossing Structures. In: VAN DER REE, R.; SMITH, D.J.; GRILO, C. (Orgs) Handbook of Road Ecology. John Wiley \& Sons, Ltd, Chichester, UK. doi: 10.1002/9781118568170.ch20, 2015.

VIEIRA, E.M. Highway mortality of mammals in Central Brazil. Ciência e Cultura, v. 48, n, 4, p. 270-272, 1996.

WEBER, M.M.; ROMAN, C.; CÁCERES, N.C. Mamíferos do Rio Grande do Sul. Santa Maria: Editora da UFSM. 2013, 556p. 